\title{
Simulation and measurement of the fractional particle number in one-dimensional optical lattices
}

\author{
Dan-Wei Zhang, ${ }^{1, *}$ Feng Mei, ${ }^{2}$ Zheng-Yuan Xue, ${ }^{1,3}$ Shi-Liang Zhu, ${ }^{2,4, \dagger}$ and Z. D. Wang ${ }^{1, \ddagger}$ \\ ${ }^{1}$ Department of Physics, Center of Theoretical and Computational Physics, The University of Hong Kong, Pokfulam Road, Hong Kong, China \\ ${ }^{2}$ National Laboratory of Solid State Microstructures, School of Physics, Nanjing University, Nanjing 210093, China \\ ${ }^{3}$ Guangdong Provincial Key Laboratory of Quantum Engineering and Quantum Materials, SPTE, South China Normal University, \\ Guangzhou 510006, China \\ ${ }^{4}$ Synergetic Innovation Center of Quantum Information and Quantum Physics, University of Science and Technology of China, \\ Hefei 230026, China
}

(Received 30 January 2015; published 10 July 2015)

\begin{abstract}
We propose a scheme to mimic and directly measure the fractional particle number in a generalized SuSchrieffer-Heeger model with ultracold fermions in one-dimensional optical lattices. We show that the fractional particle number in this model can be simulated in the momentum-time parameter space in terms of Berry curvature without a spatial domain wall. In this simulation, a hopping modulation is adiabatically tuned to form a kink-type configuration, and the induced current plays the role of an analogous soliton distributing in the time domain such that the mimicked fractional particle number is expressed by the particle transport. Two feasible experimental setups of optical lattices for realizing the required Su-Schrieffer-Heeger Hamiltonian with tunable parameters and time-varying hopping modulation are presented. We also show practical methods for measuring the particle transport in the proposed cold atom systems by numerically calculating the shift in the Wannier center and the center of mass of an atomic cloud.
\end{abstract}

DOI: 10.1103/PhysRevA.92.013612

PACS number(s): 03.75.Ss, 03.67.Ac, 03.65.Vf

\section{INTRODUCTION}

Particle fractionalization has been recognized as a remarkable and fundamental phenomenon in both relativistic quantum field theory and condensed-matter systems [1-10]. The first physical demonstration of fractionalization is the celebrated Su-Schrieffer-Heeger (SSH) model [3] of onedimensional (1D) dimerized polymers, such as polyacetylene. In this model, a kink domain wall in the electron hopping configuration induces a zero-energy soliton state carrying a half-charge $[3,4]$. The basic physics of fractionalization in the SSH model is governed by a low-energy effective Dirac Hamiltonian with topologically nontrivial background fields, which was first proposed by Jackiw and Rebbi [1]. Subsequent achievements were made to generalize the original SSH model to exhibit an irrational (arbitrary) fermion number by breaking the conjugation symmetry [11-13]. The fractional particle number (FPN) in these systems can be understood in terms of global deformations of the hole sea (or the valence band) due to the nontrivial background fields.

The SSH model has achieved great success in describing transport properties of polymers, and some novel phenomena associated with the topological solitons have also been explored in experiments [4]. In these materials, two spin orientations are present for each electron, and thus a domain wall in the polyacetylene carries an integer charge [4]. Inspired by the newly discovered quantum spin Hall insulators [14], it was theoretically proposed to realize the SSH model on the edge of this two-dimensional (2D) insulator by bringing a magnetic domain wall there, and the edge electrons with the inherent chiral symmetry may provide a direct signature of the

\footnotetext{
*zdanwei@hku.hk

†slzhu@nju.edu.cn

${ }^{\ddagger}$ zwang@hku.hk
}

FPN $[15,16]$. However, creation of such a magnetic domain wall acting only on the edge elections is experimentally challenging, and the proposed schemes are yet to be demonstrated.

In the past years, a lot of theoretical and experimental work has been carried out to simulate the Dirac equation and the involved exotic effects by using ultracold atoms [17-25]. Especially, it has been proposed to realize the (generalized) SSH model associated with the effective Dirac Hamiltonian using ultracold atomic gases in the continuum [26] and in optical lattices [27-30]. The detection of the FPN in these cold atom systems was also suggested by an optical image of the density distribution of soliton modes [26,30]. Since singlecomponent fermionic gases or component-dependent optical lattices are used in the realization of the FPN in atomic systems, the spin-doubling problem encountered in condensed-matter systems can be avoided. In a recent experiment with a 1D optical superlattice, the SSH model in the absence of spatial domain walls was realized, and its topological features were also probed [31], making direct measurement of the FPN in optical lattices to be feasible and timely.

In this paper, we propose a scheme to mimic and directly measure the FPN in the generalized SSH model using ultracold fermions in 1D optical lattices. First, we show that the FPN in this model can be simulated in the momentum-time parameter space in terms of Berry curvature without creating a spatial domain wall. In this simulation, a hopping modulation is adiabatically tuned to form a kink-type configuration, and the induced current plays the role of an analogous soliton distributing in the time domain so that the mimicked FPN in parameter space is expressed by the adiabatic particle transport. Furthermore, we explore how to implement this scheme with ultracold fermions in 1D optical lattices. We propose two experimentally setups to realize the required SSH Hamiltonian with tunable parameters and hopping modulations and then show practical methods for measuring the particle transport in the proposed cold atom systems by numerically calculating 
the shift in the Wannier center and the center of mass of an atomic cloud. Some possible concerns in realistic experiments, such as the energy scales, the adiabatic condition, and the effects of an external harmonic trap, are also considered. In comparison with the previous proposals of realizing and detecting the FPN [15,26-30], one advantage of the presented scheme is that it does not require the spatial domain in the hopping configuration, which is usually hard to create. Another advantage is the adiabatic particle transport corresponding to the value of the FPN can be directly measured in our proposed cold atom systems.

The rest of this paper is organized as follows: Section II presents a brief review on fractionlization in a generalized SSH model. In Sec. III, we elaborate our scheme of simulation and measurement of the FPN in this model, based on the Berry curvature and adiabatic transport approaches. In Sec. IV, we propose two feasible experimental setups of 1D optical lattices to realize the required Hamiltonian and then discuss how to measure the atomic particle transport in the proposed systems. Finally, a short conclusion is given in Sec. V.

\section{FRACTIONALIZATION IN THE SSH MODEL}

Before describing our scheme, we briefly review the arbitrary FPN in the generalized SSH model in this section. We start with this model described by a tight-binding Hamiltonian [7,12],

$$
H=\sum_{n}\left[J+(-1)^{n} \delta\right]\left(\hat{c}_{n}^{\dagger} \hat{c}_{n+1}+\text { H.c. }\right)+\frac{\Delta}{2} \sum_{n}(-1)^{n} \hat{c}_{n}^{\dagger} \hat{c}_{n},
$$

where $\hat{c}_{n}\left(\hat{c}_{n}^{\dagger}\right)$ is the fermion annihilation (creation) operator in site $n, J$ is the uniform hopping amplitude, $\delta$ is the dimerized hopping modulation, and $\Delta$ is a staggered potential breaking the inversion symmetry [the conjugation symmetry in the lowenergy Dirac Hamiltonian (3)]. In this lattice system as shown in Fig. 1(a), the even and odd number sites form two sublattices with modulating hopping amplitudes (on-site energies), and thus a unit cell contains two nearest lattice sites, which are used to constitute a pseudospin. When the inversion (conjugation) symmetry preserves with $\Delta=0$, the system corresponds to the original SSH model of polyacetylene.

By employing Fourier transformation in the spin basis, we can obtain the Bloch Hamiltonian of the model as

$$
\mathcal{H}=\vec{d}(k) \cdot \vec{\sigma}
$$

where $\vec{\sigma}=\left(\sigma_{x}, \sigma_{y}, \sigma_{z}\right)$ are the Pauli matrices acting on the pseudospin, $\vec{d}(k)=\left(J \cos \frac{k a}{2},-\delta \sin \frac{k a}{2}, \Delta\right)$ is the band vector with $a$ being the lattice spacing as shown in Fig. 1(a). By linearizing the Bloch bands near the Dirac point $k_{D}=\pi / a$, Hamiltonian (2) can be transformed into an effective lowenergy relativistic Hamiltonian [1,2,11],

$$
H_{D}=v_{F} \hat{p}_{x} \sigma_{x}-2 \delta \sigma_{y}+\frac{\Delta}{2} \sigma_{z},
$$

in the continuum, where $v_{F}=J a / \hbar$ is the Fermi velocity, $\hat{p}_{x}$ is the momentum operator measured from the Dirac point, and $\delta$ and $\Delta$ act as two background fields [11].
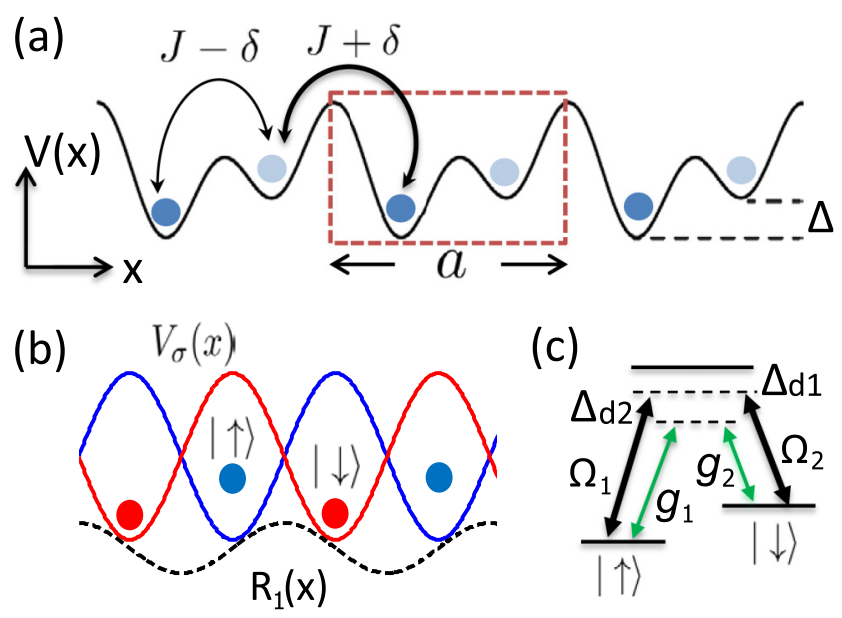

FIG. 1. (Color online) Two experimental setups for simulating the SSH Hamiltonian in 1D optical lattices. (a) A double-well optical superlattice trapping noninteracting single-component fermionic atoms. A unit cell contains two nearest lattice sites with energy offset $\Delta$ (for atoms denoted by dark and light blue small balls), and the atomic hopping exhibits staggered modulation configuration. (b) A state-dependent optical lattice trapping noninteracting twocomponent fermionic atoms where atomic states $|\uparrow\rangle$ and $|\downarrow\rangle$ are denoted by blue and red small balls. The block dotted line represents a Raman field $R_{1}(x)$. (c) Raman-assisted tunneling. The uniform atomic hopping between the nearest neighbors in the state-dependent optical lattice is realized by a large-detuned Raman transition with detuning $\Delta_{d 1}$ and Rabi frequencies of the Raman beams $\Omega_{1,2}$, whereas the hopping modulation is realized by another pair of Raman beams $g_{1,2}$ with large detuning $\Delta_{d 2}$. The Zeeman splitting gives rise to the $\Delta$ term in this system.

It has been widely studied that for a kink-type background potential with $\delta(x \rightarrow \pm \infty)= \pm \delta_{0}$, an unpaired soliton state appears at the kink-carrying FPN [2,11],

$$
\mathcal{N}_{s}=-\frac{1}{\pi} \arctan \left(\frac{4 \delta_{0}}{\Delta}\right)
$$

which may exhibit arbitrary fractional eigenvalues. The minus sign in the FPN is due to the fact that the physical fermion number in the soliton sector is defined as being measured relative to the free sector without the kink background, and this fractional part of the fermion number actually comes from the global contribution (polarization) of the valence band $[2,11,26]$. In addition, it has a topological character in the sense that it is dependent only on the asymptotic behavior of the background fields instead of their local profiles. When $\Delta \rightarrow 0$, it recovers to the half-fermion number $\pm \frac{1}{2}$ for the zero-energy soliton mode in the original SSH model [1].

It is interesting to note that fractionalization also exhibits in many low-dimensional correlated electron systems. For instance, a well-known example is that of fractional excitations in the fractional quantum Hall regime [5,9], which is a consequence of strong Coulomb interaction among 2D electrons in partially filled Landau levels. In addition, the collective excitations in some 1D interacting fermion systems may be characterized by effective fractional charges via the spin-charge separation mechanism [10]. Fractionalization in these systems is basically due to electron correlations and 
hence is completely different from that in the SSH model of noninteracting fermions. The fractional charges in some correlated electron systems have been directly observed in experiments $[9,10]$. However, despite the fractionalization in SSH model being investigated for decades, the FPN there is yet to be directly measured in solid-state materials (mostly due to the spin-doubling problem) or in artificial systems, even for the simplest half-fermion-number case with $\Delta=0$. Therefore, experimentally feasible schemes for direct measurement of the intrinsic FPN in the SSH model would be of great value.

\section{SCHEME TO DIRECTLY MEASURE THE FPN}

Several schemes have been proposed to realize the SSH model and to probe the soliton modes with the FPN in cold atom systems [27-30]; however, the realization of the required kink background and the local detection of the fermion number of a soliton state therein are still challenging in practical experiments. In the following, we propose a simpler scheme to mimic and then directly measure the FPN via the adiabatic particle transport.

The energy bands of the SSH model described by Hamiltonian (2) can be mapped onto a two-level system in the Bloch sphere with the parametrized Bloch vector $\vec{S}=h_{0}(\sin \theta \cos \phi, \sin \theta \sin \phi, \cos \theta)$, where $\theta$ and $\phi$ are the polar and azimuthal angles, respectively. In this mapping, we have

$$
\begin{aligned}
h_{0} & =\sqrt{4 J^{2} \cos ^{2}(k a / 2)+4 \delta^{2} \sin ^{2}(k a / 2)+\Delta^{2} / 4}, \\
\theta & =\arccos \left(\Delta / 2 h_{0}\right), \\
\phi & =-\arctan \left[\frac{\delta \sin (k a / 2)}{J \cos (k a / 2)}\right] .
\end{aligned}
$$

The degeneracy point locates at $k=\pi / a, \delta=0$, and $\Delta=$ 0 . We consider the fractionalization in this band insulator at half-filling [26], corresponding to the low-energy level with the eigenstate $\left|u_{-}\right\rangle=\left(\sin \frac{\theta}{2} e^{-i \phi},-\cos \frac{\theta}{2}\right)^{\mathrm{T}}$ with superscript $\mathrm{T}$ being the transposition of matrix. In this framework, one can define the Berry connection $A_{\theta}=\left\langle u_{-} \mid i \partial_{\theta} u_{-}\right\rangle=0$ and $A_{\phi}=\left\langle u_{-} \mid i \partial_{\phi} u_{-}\right\rangle=\sin ^{2} \frac{\theta}{2}$.

Instead of considering the kink background field in the spatial domain, here we introduce a time-varying hopping modulation with a kink-type ramping configuration,

$$
\delta(t)=\delta_{0} \tanh \left[\xi\left(t-t_{0}\right)\right],
$$

where $t_{0}$ denotes the center of the time domain wall and $\xi$ represents the ramp frequency. We assume $t_{0} \gg 1 / \xi$ (actually $t_{0}=5 / \xi$ is large enough) such that $\delta(0) \simeq-\delta_{0}$ at the beginning $t=0$ and then adiabatically ramp the system to $t=t_{f}=2 t_{0}$ with $\delta\left(t_{f}\right) \simeq \delta_{0}$ at the end. That is to say, we simulate a kink potential in the time domain instead of creating it in real space. In this dynamical case, the bulk gap is $E_{g}=2 \sqrt{\Delta^{2}+16 \delta(t)^{2}}$, which will close at $t=t_{0}$ for the original SSH model with $\Delta=0$. To guarantee the adiabatic condition for the original SSH case, which requires a gapped bulk band, we can use a time-varying staggered potential with the form $\Delta(t)=\delta_{0} \sin \left(\pi t / 2 t_{0}\right)$.

We consider the adiabatic evolution of the system with the ramping parameter $\delta(t)$ [and $\Delta(t)$ for the original $\mathrm{SSH}$ case] and provide that the Fermi level lies inside the band gap in the whole progression. The Berry phase effect of this 1D band insulator can be measured from the particle transport [7]. This is an analog of the adiabatic charge pumping proposed by Thouless [32]; however, the parametric driving in our case does not form a closed cycle but only a half one. The topological pumping in cold atom systems and photonic quasicrystals have been discussed in the contexts of the SSH model [33-35] and the 1D quasiperiodic Harper model [36-40] where the pumping particle is shown to be quantized one over one period and can be fractional over a fraction of one period [40].

In the momentum-time $(k-t)$ parameter space, we can rewrite the Berry connection as $A_{k}=\partial_{k} \phi A_{\phi}+\partial_{k} \theta A_{\theta}$ and $A_{t}=\partial_{t} \phi A_{\phi}+\partial_{t} \theta A_{\theta}$. Thus the Berry curvature $\mathcal{F}_{k t}=$ $\partial_{k} A_{t}-\partial_{t} A_{k}$ in the $k-t$ space can be obtained as

$$
\mathcal{F}_{k t}=\frac{\Delta J \sin ^{2} \frac{k a}{2} \partial_{t} \delta(t)}{2\left[4 J^{2} \cos ^{2} \frac{k a}{2}+4 \delta(t)^{2} \sin ^{2} \frac{k a}{2}+\Delta^{2} / 4\right]^{3 / 2}},
$$

where $J$ and $\Delta$ are assumed to be constants here. We note that Berry curvature distribution given by Eq. (7) is modified for time-varying $\Delta(t)=\delta_{0} \sin \left(\pi t / 2 t_{0}\right)$ discussed previously, however, the corresponding FPN given by the following Eq. (8) remains since it just depends on the boundaries of the background fields [4].

Figure 2(a) shows an example of the Berry curvature distribution in the center region of the $k-t$ space for typical parameters, whereas the Berry curvature outside is almost vanishing. In the small $\Delta$ limit, a sharp peak exhibits in the Berry curvature distribution at the position of $(k, t)=\left(\pi / a, t_{0}\right)$, which is the dominant contribution to its integration over the parameter space. Figure 2(b) shows the corresponding spin texture, which is interpreted as a mapping from the $k-t$ parameter space onto the Bloch sphere by Eq. (5). Figures 2(c) and 2(d) show the static energy spectrum and the adiabatic density variation in each lattice site with respect to the hopping modulation for the lattice size $L=100$, respectively. It looks like a density kink-soliton (antisoliton) configuration appears in the time domain.

The particle transport for the ground band in this 1D band insulator over the ramping progression of parameter $\delta(t)$ from $t=0$ to $t=t_{f}$ is given by the integration of the Berry curvature,

$$
\begin{aligned}
Q & =-\frac{1}{2 \pi} \int_{0}^{t_{f}} d t \int_{0}^{2 \pi} d k \mathcal{F}_{k t} \\
& =\frac{1}{2 \pi} \int_{0}^{2 \pi} d k\left[A_{k}\left(k, t_{f}\right)-A_{k}(k, 0)\right] \\
& =-\frac{1}{\pi} \arctan \left(\frac{4 \pi J \delta_{0}}{\Delta \sqrt{\pi^{2} J^{2}+4 \delta_{0}^{2}+\Delta^{2} / 4}}\right) \\
& \simeq-\frac{1}{\pi} \arctan \left(\frac{4 \delta_{0}}{\Delta}\right)=\mathcal{N}_{s},
\end{aligned}
$$

where the approximation satisfies well for $J \gg \delta_{0}, \Delta$ and becomes exact when $\Delta=0$. In the calculation, the integration of $\partial_{k} A_{t}$ over $k$ vanishes due to the periodic condition in the Berry vector potential [7]. Here the unquantized adiabatic particle can be regarded as the polarization change in this 1D band insulator $[7,41]$, which has also been discussed in 

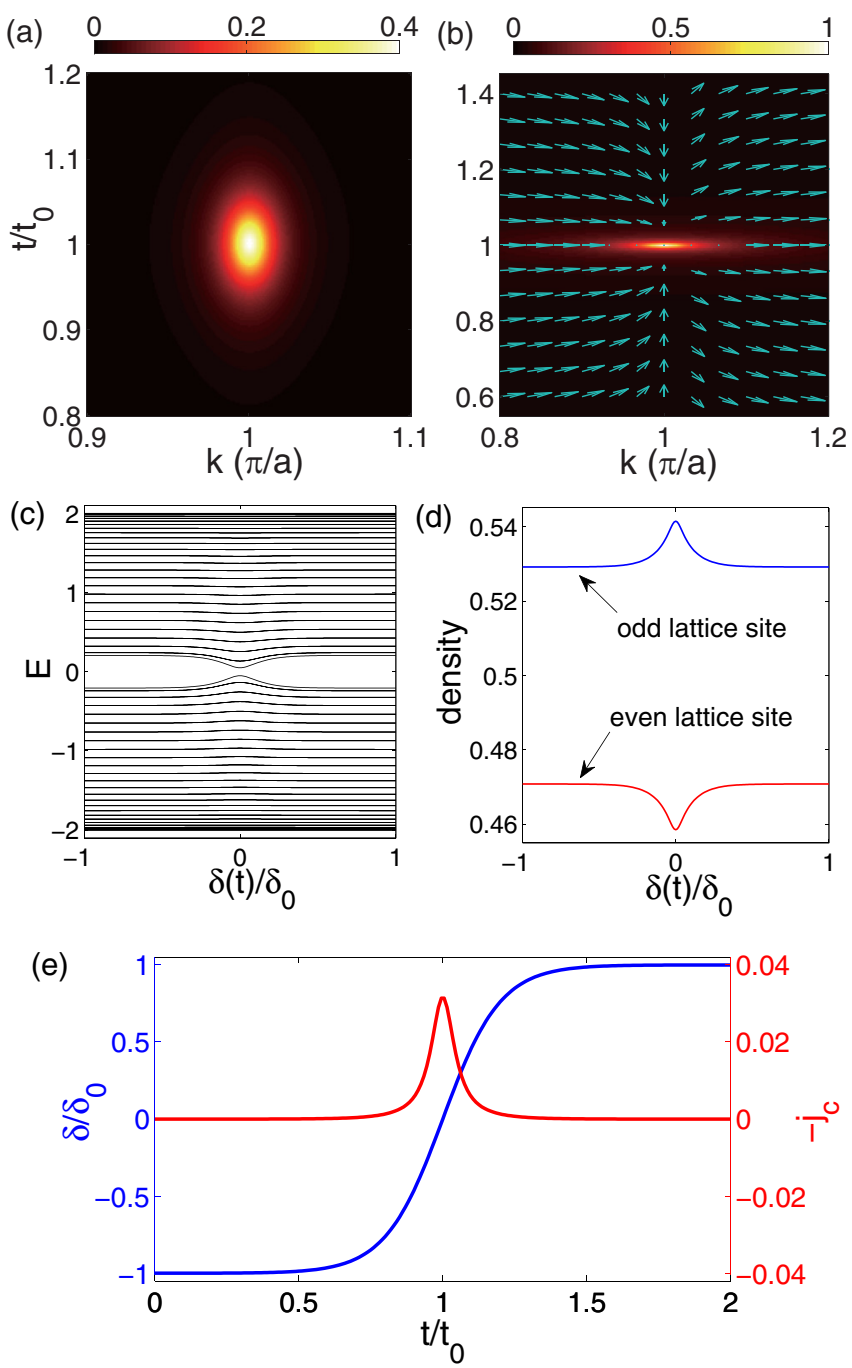

FIG. 2. (Color online) (a) Berry curvature distribution of the ground band $\mathcal{F}_{k t}$ in the center region of the $k-t$ parameter space. The Berry curvature outside is almost vanishing, and integration over the whole parameter space $\left(0 \leq k \leq 2 \pi / a, 0 \leq t \leq 2 t_{0}\right)$ gives the particle transport. (b) Spin texture of the SSH model, interpreted as a mapping from the $k$ - $t$ parameter space onto the Bloch sphere by Eq. (5). The colors show the $S_{z}$-component $\cos [\theta(k, t)]$, and the arrows show the azimuthal component $\left(S_{x}, S_{y}\right)=(\sin \theta \cos \phi, \sin \theta \sin \phi)$. Here the lengths of all the arrows have been divided by a factor $\pi$ for visibility. (c) The energy spectrum for a lattice system with size $L=100$. (d) The variation in density in each lattice site with respect to the time-varying hopping modulation at half-filling. (e) The analog of kink potential and solitonlike current in the time domain under adiabatic conditions. The parameters in (a)-(e) are $J=1, \delta_{0}=\Delta=0.1$, and $t_{0}=5 / \xi$.

the context of nanotubes and ferroelectric materials [42]. If one consider an additional anti-kink-type modulation to form a full cycle, then the particle transport will be quantized $( \pm 1$ or 0 depending on the loop of the cycle) after one period as the integration of the Berry curvature in the extensional region contributes the other fractional portion $[7,15]$.

There are actually close connections between the FPN of a soliton state and the adiabatic transport via the Berry phase approach in Eq. (8). We can consider the response equation in the progression of dynamical generation of the background field $[6,11]$,

$$
\rho_{s}=\frac{1}{2 \pi} \partial_{x} \Theta(x, t), \quad j_{c}=\frac{1}{2 \pi} \partial_{t} \Theta(x, t),
$$

where $\Theta(x, t)$ represents the generalized angular angle of the background field, $\rho_{s}$ denotes the soliton density distribution near the spatial domain wall, and $j_{c}$ is the induced current. In the present model with $J \gg \delta, \Delta$ (in which case the same polarization variation is obtained by the band Hamiltonian and the low-energy Dirac Hamiltonian), the angular angle $\Theta=$ $-\arctan (4 \delta / \Delta)$ is just time dependent. Therefore, the induced current mimics an analog of the kink soliton in the time domain as shown in Fig. 2(e). The induced current over the whole time domain gives the transferred particle,

$$
Q=\int_{0}^{t_{f}} j_{c}(t) d t,
$$

which takes the value given by Eq. (8) and depends only on the boundaries of the angular angle under the adiabatic condition.

So far, we have described our scheme to simulate the FPN in the SSH model in a parameter space and to directly measure it via the adiabatic transport. In contrast to the previous schemes [15,26-30], the presented scheme does not involve the spatial kink domain in the hopping configuration, which is usually hard to realize and (or) control in experiments. In addition, the particle transport corresponding to the value of the FPN can be directly measured in cold atom systems, such as from the measurement of atomic density distribution and atomic current [43], which will be discussed in 1D optical lattices in the next section.

\section{EXPERIMENTAL IMPLEMENTATION IN OPTICAL LATTICES}

In this section, we turn to discuss the implementation of our scheme of mimicking and measuring the FPN in 1D optical lattices. We first propose two experimental setups to realize the required SSH Hamiltonian with tunable parameters and then discuss how to measure the particle transport in the proposed cold atom systems by numerically calculating the shift in the Wannier center and the center of mass of an atomic cloud.

\section{A. Two experimental setups}

The first experimental setup we proposed is a 1D optical superlattice trapping a noninteracting atomic gas of singlecomponent fermions as shown in Fig. 1(a). Such an optical lattice has been widely realized in experiments [31,43-45]. It is generated by superimposing two lattice potentials with short and long wavelengths differing by a factor of 2 with the optical potential given by

$$
V(x)=V_{1} \sin ^{2}\left(k_{1} x+\varphi\right)+V_{2} \sin ^{2}\left(2 k_{1} x\right) .
$$

Here $k_{1}$ is the wave vector of the short-wavelength trapping lasers (the lattice spacing $\left.a=2 \pi / k_{1}\right), \varphi$ and $V_{1,2}$ are the relative phase and the strengths of the two standing waves, respectively. By varying the laser intensity and the phase, one can fully control the lattice system with ease [31,43-45] and then make the system well described by Hamiltonian (1) of 
the SSH model in the tight-binding regime [31]. In the experiments [31,43-45], the hopping configuration $J+(-1)^{j} \delta$ can be adjusted by varying potential strengths $V_{1,2}$ or swapping the relative phase $\varphi$, and the staggered potential $\Delta$ can be tuned by the phase. Therefore in this system, a straightforward way to realize the required hopping modulation with a kink-type configuration in the time domain is by changing these tunable parameters of the optical superlattice with a well-designed sequence [31].

Another experimental setup, which would be more convenient as we will see in the following, is loading an ultracold Fermi gas of two-component (internal states $|\sigma\rangle$ with $\sigma=$ $\uparrow, \downarrow)$ atoms in a state-dependent optical lattice [46]. It has been proposed to realize the SSH Hamiltonian with a spatial domain wall in this system [27], and such a state-dependent optical lattice has been experimentally created by superposing two linearly polarized laser beams with a relative polarized angle [46]. The separation and potential depth for different atomic components can be well controlled by the angle and the laser intensity with a simple example of such a 1D lattice potential as shown in Fig. 1(b),

$$
V_{\sigma}(x)=V_{0} \sin ^{2}\left(k_{s} x \pm \pi / 4\right) .
$$

Here $V_{0}$ is the lattice potential depth, $k_{s}$ is the wavelength of the laser beams (the lattice spacing $a=2 \pi / k_{s}$ ), and $\pm \pi / 4$ are the polarization angles for atomic states $|\uparrow\rangle$ and $|\downarrow\rangle$, respectively.

For sufficiently deep lattices, the atoms in the system must alter their internal states in order to tunnel between two nearest-neighbor lattice sites. This can be achieved by the Raman-assisted tunneling method [43,47-50] as shown in Fig. 1(c). The energy offset between two atomic states arises from the external Zeeman field and then plays the role of tunable parameter $\Delta$ in this system. Two pairs of Raman beams with Rabi frequencies $\Omega_{1,2}$ and $g_{1,2}$ are used to induce two large-detuned Raman transitions with detunings $\Delta_{d 1}$ and $\Delta_{d 2}$, respectively. One can use the former pair of Raman beams to realize the uniform nearest-neighbor hopping,

$$
J=A_{0} \int w_{\uparrow}^{*}\left(x-x_{n}\right) e^{i k_{x} x} w_{\downarrow}\left(x-x_{n+1}\right) d x,
$$

where $A_{0}=\left|\Omega_{1} \Omega_{2}^{*}\right| / \Delta_{d 1}$ is the effective Raman strength constant, $k_{x}$ is the momentum difference along the $x$ axis between the two beams, and $w_{\sigma}(x)$ are the Wannier functions of the lowest Bloch band for atomic state $|\sigma\rangle$.

To realize the time-varying hopping modulation term, one can use another pair of laser beams with a resulting Raman field as shown in Fig. 1(b),

$$
R_{1}=g_{1} g_{2}^{*} / \Delta_{d 2}=A_{1}(t) \sin \left(2 k_{s} x+\pi / 2\right),
$$

where $A_{1}$ is a time-dependent constant controlled by the laser intensities or detuning $\Delta_{d 2}$. In this way, the hopping modulation $(-1)^{n} \delta(t)$ is given by

$$
A_{1}(t) \int w_{\uparrow}^{*}\left(x-x_{n}\right) \sin \left(2 k_{s} x+\pi / 2\right) w_{\downarrow}\left(x-x_{n+1}\right) d x .
$$

Here the staggered hopping modulation is a consequence of the relative spatial configuration of the lattice and the Raman field: The period of $R_{1}(x)$ is double the lattice period, and $R_{1}(x)$ is antisymmetric corresponding to the center of each lattice site as shown in Fig. 1(b). Thus, to realize the proposed particle transport scheme in this system, we can adjust the Zeeman field and the Raman field $R_{0}$ to tune the parameters $\Delta$ and $J$ in the SSH Hamiltonian and then independently vary the intensity of another Raman field $A_{1}(t)$ in time with a kink-type form. The case of time-varying $\Delta(t)$ can also be achieved in a similar way. The time modulation of the Raman coupling in ultracold atoms has been demonstrated in recent experiments [51].

Considering ${ }^{40} \mathrm{~K}$ atoms and typical lattice spacing $a=$ $532 \mathrm{~nm}$, one has the recoil energy $E_{R} / \hbar \approx 30 \mathrm{kHz}$. For the optical superlattice system with an intermediately deep lattice, a typical uniform hopping strength is $J \sim 0.1 E_{R}$, and the other parameters $\delta$ and $\Delta$ can be tunable in a wide regime [31]. For the state-dependent optical lattice system, the uniform hopping strength given by Eq. (13) is proportional to the effective Raman intensity $A_{0}$, which is typically on the order of megahertz, and the overlap integral of the Wannier functions between neighbor lattice sites can be about $10^{-2}$ [52]. Thus the Raman-induced uniform hopping strength in this system is $J \sim 0.4 E_{R}$, and the nature (next-nearest-neighbor) hopping $t_{N}$ within sublattices can be effectively suppressed by sufficiently deep lattice $V_{0}$. For example, the numerical calculation shows that $t_{N} \lesssim 10^{-3} E_{R}$ for $V_{0} \approx 22 E_{R}$ [52]. We can consider typical parameters $\delta_{0}$ and $\Delta$ (the minimum bulk gap in the dynamical progression is $2 \Delta$ ) on the order of $0.1 J$. In this case, the adiabatic approximation works well for the ramp time $t_{f} \gg \hbar / 0.1 J \sim 1 \mathrm{~ms}$. Thus one can choose the ramp frequency $\xi=0.01 \mathrm{~J} / \hbar$ and $t_{0}=50 \mathrm{~ms}$, which is well shorter than the typical coherence time in cold atom experiments. The nonadiabatic Landau-Zener transition from the ground band to the excited band for the chosen parameters is then given by $P_{\mathrm{LZ}} \approx e^{-\pi(0.1 J)^{2} / 4 \hbar \delta_{0} \xi} \approx 0$. In addition, the finite temperature effects do not interfere with the particle transport progression for temperatures lower than the energy gap [33]. This requires a temperature on the order of $0.08 E_{R} / k_{B} \sim 20 \mathrm{nK}\left(k_{B}\right.$ is the Boltzmann constant), which has been achieved in current experiments with, e.g., ${ }^{40} \mathrm{~K}$ atoms. So we can conclude that the required Hamiltonian with tunable parameters and the adiabatic condition are able to be realized under realistic circumstances.

\section{B. Experimental measurement methods}

It has been shown that the particle transport can be connected with the Wannier center based on the modern theory of charge polarization [7,41]. Especially, the shift in the Wannier center in each unit cell is

$$
X_{d}=x_{c}\left(t=t_{f}\right)-x_{c}(t=0),
$$

where $x_{c} \equiv\left\langle w_{n}|\hat{x}| w_{n}\right\rangle$ is the Wannier center with $\left|w_{n}(x)\right\rangle=$ $\frac{1}{2 \pi} \int_{-\pi}^{\pi} e^{-i k(n-x)}\left|u_{-}(k)\right\rangle$ being the Wannier function of the ground band in the $n$th unit cell. The shift in the Wannier center encodes the adiabatic particle transport (the variation in polarization) as $[33,39,40]$

$$
X_{d} / a=Q .
$$

In Fig. 3, we have numerically calculated the variation in the Wannier center in each lattice site in the proposed system. For the symmetric case with $\Delta=0^{+}$(the solid blue line) and $\Delta(t)=\delta_{0} \sin \left(\pi t / 2 t_{0}\right)$ (the dashed red line) shown in Fig. 3(a), after adiabatically tuning the hopping modulation over the kink 

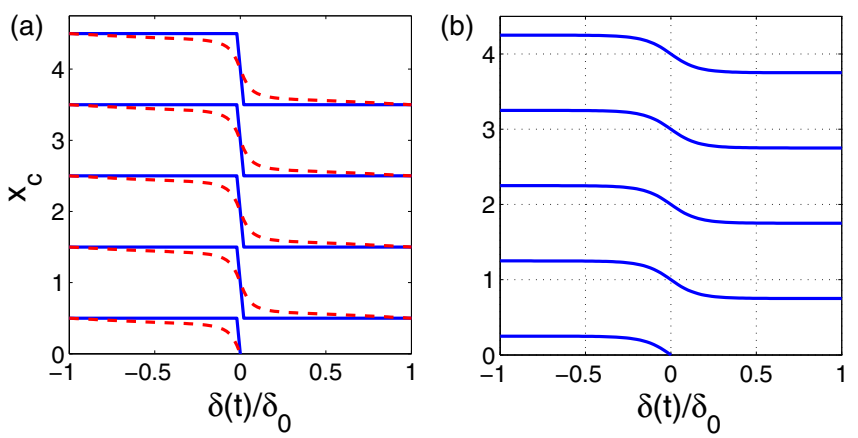

FIG. 3. (Color online) The shift in the Wannier center in each lattice as a response to the adiabatically tuning hopping modulation. (a) The symmetric case with $\Delta=0^{+}$(solid blue line) and $\Delta(t)=$ $\delta_{0} \sin \left(\pi t / 2 t_{0}\right)$ (dashed red line) with $\delta_{0}=0.1 J$. After tuning the hopping modulation over the kink form, the Wannier center for each lattice site shifts downwards one site, i.e., one-half of the unit cell, corresponding to $Q=-1 / 2$ as expected for the half-charge in the original SSH model. (b) The symmetry-breaking case with $\Delta=4 \delta_{0}=0.1 \mathrm{~J}$, the shift in the Wannier center is nearly half a lattice site, corresponding to $Q=-1 / 4$ as expected in this case. Other parameters in (a) and (b) are $J=1$ and $t_{0}=5 / \xi$.

form, the Wannier center in each lattice site shifts downwards one site, that is, exactly one-half of the unit cell. According to Eqs. (8) and (16), the adiabatic particle transport is $Q=-1 / 2$ (the sign depends on the shift direction), as expected for the half-charge in the original SSH model. For the symmetrybreaking case with $\Delta=4 \delta_{0}=0.1 J$ as shown in Fig. 3(b), the shift in the Wannier center is nearly half a lattice site, which is consistent with the expected $Q=-1 / 4$ in this case.

The shift in Wannier center shown in Fig. 3 implies the appearance of atomic current (the transport of particles) in each unit cell, which flows through the whole lattice system. Under the adiabatic condition, the atomic current will take the solitonic form in the ramping progression, which is similar to the example shown in Fig. 2(e). In principle, the transport dynamics can be detected by using the single-atom in situ imaging technology in optical lattices [53]. Thus, the variation in atomic density distribution in a unit cell associated with induced current can be experimentally extracted out in this way. In our proposed systems, it would be more convenient to detect the global current through the whole lattice by measuring the time evolution of the atom fractions in the even and odd sublattices, instead of using in situ detection in a single unit cell. For the double-well superlattice system in Fig. 1(a), the atomic current associated with the atom fractions of the even or odd sublattices has been measured in the experiment by transferring the atoms to higher-lying Bloch bands and applying a subsequent band mapping technique [43]. For the state-dependent optical lattice system in Fig. 1(b), the even and odd sublattices trap $|\uparrow\rangle$ and $\downarrow\rangle$ atoms, respectively. Therefore in this system, one can simply measure the evolution of atom fractions via optical imaging of the up-component (downcomponent) atoms, such as using state-resolved time-of-flight measurements [54]. Therefore, one can obtain the particle transport $Q$ by its integration over the time domain as given by Eq. (10), which corresponds to the mimicked FPN in this system.
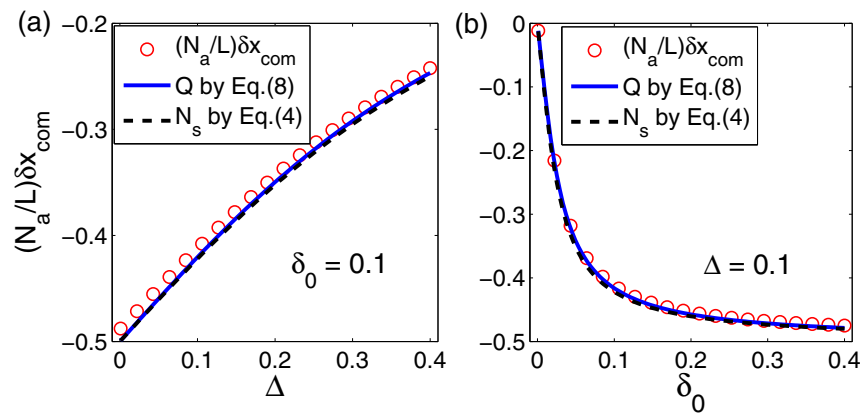

FIG. 4. (Color online) The shift in the center of mass of an atomic cloud as a function of (a) the parameter $\Delta$ with fixed $\delta_{0}=0.1$ and (b) the parameter $\delta_{0}$ with fixed $\Delta=0.1$ for a finite lattice system with $L=400$ sites and at half-filling $N_{a} / L=1 / 2$, respectively. The solid (blue) line and the dashed (black) line denote the corresponding particle transport $Q$ obtained by Eq. (8) and fractional particle number $\mathcal{N}_{s}$ obtained by Eq. (4), respectively, in the text. Other parameters in (a) and (b) are $J=1$ and $t_{0}=5 / \xi$.

In addition, the particle transport can be directly measured from the shift in the center of mass of an atomic cloud in a finite lattice system $[33,35,40]$. In our system, the center of mass of an atomic cloud in the lattice with $L$ sites $x_{\text {com }}(t)$ is given by

$$
x_{\mathrm{com}}(t)=\frac{1}{N_{a}} \sum_{n=1}^{L} \sum_{\varepsilon_{o c}}\left|\psi_{o c}(n, t)\right|^{2} n,
$$

where $N_{a}=L / 2$ is the atomic number at half-filling, $\varepsilon_{o c}$ denotes the occupied state of the fermionic atoms, and $\psi_{o c}$ is the corresponding wave function. Under the adiabatic evolution with $\delta(0) \rightarrow \delta\left(t_{f}\right)$ described by Eq. (6), the center of mass of the system shifts from the position $x_{\text {com }}(0)$ to $x_{\text {com }}\left(t_{f}\right)$. It can be proved that the shift in the center-of-mass $\delta x_{\mathrm{com}}=$ $x_{\text {com }}\left(t_{f}\right)-x_{\text {com }}(0)$ is proportional to the particle transport in the infinite $L$ limit $[33,40]$,

$$
\frac{N_{a}}{L} \delta x_{\text {com }}=Q .
$$

If $L$ is large enough such that the bulk properties of the system are almost not affected by the edges, $N_{a} \delta x_{\text {com }} / L$ in the above equation will be approximated to be the ideal particle transport $Q$ in infinite systems. In Fig. 4, we have calculated the shift in the center of mass of an atomic cloud for a lattice system with $L=400$ sites, $N_{a} \delta x_{\text {com }} / L$ (the red circles) as a function of the parameters $\Delta$ [Fig. 4(a)] and $\delta_{0}$ [Fig. 4(b)], respectively. As shown in Figs. 4(a) and 4(b), the calculated shift in the center of mass is well described by the particle transport $Q$ (the solid blue line) obtained by Eq. (8) with small deviations. These deviations are due to the finite-size effects and become smaller and smaller with the increase in the lattice size in our simulations, which will be invisible in practical experiments. For comparison, we also plot the mimicked FPN $\mathcal{N}_{s}$ given by Eq. (4) in this system in Fig. 4 (the dashed black line). From Fig. 4, one can see that the corresponding FPN is nearly equal to the particle transport within the parameter regimes. In current experiments, the center-of-mass position of an atomic cloud can be directly and precisely measured, either by using an in situ measurement of the atomic density distribution in the 
optical lattices [53] or by being deduced from the time-of-flight imaging [54].

Finally, we note that a shallow-enough harmonic trap in practical experiments will not affect the particle transport $[33,39]$ and the main results of this paper remain intact. In order to take the effect of the harmonic trap into account, we can add a term $H_{t}=V_{t} \sum_{n}(n-L / 2)^{2} \hat{c}_{n}^{\dagger} \hat{c}_{n}$ into Hamiltonian (1), where $V_{t}$ is the trap strength and $L$ is the lattice size. Within a local-density approximation, the lower band will still be filled at the center of the trap, and thus the shift in the Wannier center in these lattice sites is expected to be nearly the same as those shown in Fig. 3, whereas the band is only partially filled near the edge with the local trap energy $V_{t}(n-L / 2)^{2} \gtrsim E_{g}$ such that the pumping argument does not apply to this region [33]. Therefore in practical experiments, one may emphasize the shift in the Wannier center in the central region or turn the trap strength to a small value $V_{t} \sim 4 E_{g} / L^{2}$. For the shift in the center of mass of an atomic cloud, our numerical simulations demonstrate that the results shown in Fig. 4 preserve with a deviation less than $2 \%$ for $V_{t} \approx 0.6 \times 10^{-5} \mathrm{~J}$, whereas $V_{t} \approx 10^{-4} \mathrm{~J}$ is enough if the lattice size reduces to $L=100$, which are consistent with the estimates in the local-density analysis.

\section{CONCLUSIONS}

To summarize, we have proposed a scheme to mimic and measure the FPN in the generalized SSH model with cold fermions in 1D optical lattices. It has been shown that the FPN in this model can be simulated in the momentum-time parameter space in terms of Berry curvature without a spatial domain wall. In this simulation, a hopping modulation is adiabatically tuned to form a kink-type configuration, and the induced current plays the role of a soliton in the time domain, so the FPN is expressed by the particle transport. We have also proposed two experimental setups of optical lattices to realize the required Hamiltonian with tunable and time-varying parameters and considered the energy scales and the adiabatic condition under practical circumstances. Finally we have discussed how to directly measure the particle transport in the proposed systems by numerically calculating the shift in the Wannier center and the center of mass of an atomic cloud. Considering that all the ingredients to implement our scheme in optical lattices have been achieved in the recent experiments, it is anticipated that the presented proposal will be tested in an experiment in the near future. The direct measurement of such a mimicked FPN in cold atom experiments will be an important step toward exploring fractionalization and topological states in cold atom systems. Extensions of this paper can enable simulating and measuring the FPN emerging in two- and threedimensional Dirac Hamiltonians with topologically nontrivial (vortex and monopole) background fields [1,8], which has been theoretically studied but remains elusive in nature. It will be also interesting to simulate a variety of topological states [6] and study their properties in the parameter space using cold atoms.

\section{ACKNOWLEDGMENTS}

This work was supported by the RGC of Hong Kong (Grants No. HKU7045/13P and No. HKU173051/14P), the SKPBR of China (Grants No. 2011CB922104 and No. 2013CB921804), the NSFC (Grants No. 11125417 and No. 11474153), and the PCSIRT (Grant No. IRT1243).
[1] R. Jackiw and C. Rebbi, Phys. Rev. D 13, 3398 (1976).

[2] A. Niemi and G. Semenoff, Phys. Rep. 135, 99 (1986).

[3] W. P. Su, J. R. Schrieffer, and A. J. Heeger, Phys. Rev. Lett. 42, 1698 (1979); Phys. Rev. B 22, 2099 (1980).

[4] A. J. Heeger, S. Kivelson, J. R. Schrieffer, and W. P. Su, Rev. Mod. Phys. 60, 781 (1988).

[5] R. B. Laughin, H. Störmer, and D. Tsui, Rev. Mod. Phys. 71, 863 (1988).

[6] X.-L. Qi, T. L. Hughes, and S.-C. Zhang, Phys. Rev. B 78, 195424 (2008).

[7] D. Xiao, M.-C. Chang, and Q. Niu, Rev. Mod. Phys. 82, 1959 (2010).

[8] C.-Y. Hou, C. Chamon, and C. Mudry, Phys. Rev. Lett. 98, 186809 (2007); B. Seradjeh, C. Weeks, and M. Franz, Phys. Rev. B 77, 033104 (2008); A. Rüegg and G. A. Fiete, ibid. 83, 165118 (2011).

[9] R. de-Picciotto, M. Reznikov, M. Heiblum, V. Umansky, G. Bunin, and D. Mahalu, Nature (London) 389, 162 (1997).

[10] H. Steinberg, G. Barak, A. Yacoby, L. N. Pfeiffer, K. W. West, B. I. Halperin, and K. L. Hur, Nat. Phys. 4, 116 (2008); H. Kamata, N. Kumada, M. Hashisaka, K. Muraki, and T. Fujisawa, Nat. Nanotechnol. 9, 177 (2014).

[11] J. Goldstone and F. Wilczek, Phys. Rev. Lett. 47, 986 (1981); H. Takayama, Y. R. Lin-Liu, and K. Maki, Phys. Rev. B 21, 2388 (1980).
[12] M. J. Rice and E. J. Mele, Phys. Rev. Lett. 49, 1455 (1982).

[13] R. Jackiw and G. Semenoff, Phys. Rev. Lett. 50, 439 (1983).

[14] M. Z. Hasan and C. L. Kane, Rev. Mod. Phys. 82, 3045 (2010); X. L. Qi and S. C. Zhang, ibid. 83, 1057 (2011).

[15] X.-L. Qi, T. L. Hughes, and S.-C. Zhang, Nat. Phys. 4, 273 (2008).

[16] J. I. Väyrynen and T. Ojanen, Phys. Rev. Lett. 107, 166804 (2011).

[17] S.-L. Zhu, B. Wang, and L.-M. Duan, Phys. Rev. Lett. 98, 260402 (2007); C. Wu, ibid. 101, 186807 (2008); L.-K. Lim, C. M. Smith, and A. Hemmerich, ibid. 100, 130402 (2008); J.-M. Hou, W.-X. Yang, and X.-J. Liu, Phys. Rev. A 79, 043621 (2009); K. L. Lee, B. Gremaud, R. Han, B. G. Englert, and C. Miniatura, ibid. 80, 043411 (2009); A. Bermudez, N. Goldman, A. Kubasiak, M. Lewenstein, and M. A. Martin-Delgado, New J. Phys. 12, 033041 (2010).

[18] N. Goldman, A. Kubasiak, A. Bermudez, P. Gaspard, M. Lewenstein, and M. A. Martin-Delgado, Phys. Rev. Lett. 103, 035301 (2009); A. Bermudez, L. Mazza, M. Rizzi, N. Goldman, M. Lewenstein, and M. A. Martin-Delgado, ibid. 105, 190404 (2010)

[19] J. Y. Vaishnav and C. W. Clark, Phys. Rev. Lett. 100, 153002 (2008); G. Juzeliūnas, J. Ruseckas, M. Lindberg, L. Santos, and P. Öhberg, Phys. Rev. A 77, 011802(R) (2008); Y. Zhang, L. Mao, and C. Zhang, Phys. Rev. Lett. 108, 035302 (2012); S. 
Cao, D.-W. Zhang, H. Yan, and Z.-Y. Xue, JETP Lett. 98, 858 (2014).

[20] M. Merkl, A. Jacob, F. E. Zimmer, P. Öhberg, and L. Santos, Phys. Rev. Lett. 104, 073603 (2010); S.-L. Zhu, D.-W. Zhang, and Z. D. Wang, ibid. 102, 210403 (2009); D.-W. Zhang, Z.-Y. Xue, H. Yan, Z. D. Wang, and S.-L. Zhu, Phys. Rev. A 85, 013628 (2012).

[21] See the reviews, D.-W. Zhang, Z. D. Wang, and S.-L. Zhu, Front. Phys. 7, 31 (2012); F. Mei, D.-W. Zhang, and S.-L. Zhu, Chin. Phys. B 22, 116106 (2013).

[22] T. Salger, C. Grossert, S. Kling, and M. Weitz, Phys. Rev. Lett. 107, 240401 (2011).

[23] L. Tarruell, D. Greif, T. Uehlinger, G. Jotzu, and T. Esslinger, Nature (London) 483, 302 (2012).

[24] L. J. LeBlanc, M. C. Beeler, K. Jimenez-Garcia, A. R. Perry, S. Sugawa, R. A. Williams, and I. B. Spielman, New J. Phys. 15, 073011 (2013).

[25] C. Qu, C. Hamner, M. Gong, C. Zhang, and P. Engels, Phys. Rev. A 88, 021604 (2013).

[26] D.-W. Zhang, L.-B. Shao, Z.-Y. Xue, H. Yan, Z. D. Wang, and S.-L. Zhu, Phys. Rev. A 86, 063616 (2012).

[27] J. Ruostekoski, G. V. Dunne, and J. Javanainen, Phys. Rev. Lett. 88, 180401 (2002); J. Ruostekoski, J. Javanainen, and G. V. Dunne, Phys. Rev. A 77, 013603 (2008).

[28] X.-J. Liu, Z.-X. Liu, and M. Cheng, Phys. Rev. Lett. 110, 076401 (2013).

[29] X. Li, E. Zhao, and W. V. Liu, Nat. Commun. 4, 1523 (2013).

[30] J. Javanainen and J. Ruostekoski, Phys. Rev. Lett. 91, 150404 (2003).

[31] M. Atala, M. Aidelsburger, J. T. Barreiro, D. Abanin, T. Kitagawa, E. Demler, and I. Bloch, Nat. Phys. 9, 795 (2013).

[32] D. J. Thouless, Phys. Rev. B 27, 6083 (1983).

[33] L. Wang, M. Troyer, and X. Dai, Phys. Rev. Lett. 111, 026802 (2013).

[34] H. Guo, Phys. Lett. A 378, 1316 (2014); arXiv:1311.4134.

[35] Y. Qian, M. Gong, and C. Zhang, Phys. Rev. A 84, 013608 (2011).

[36] P. G. Harper, Proc. Phys. Soc. London, Sect. A 68, 874 (1955); S. Aubry and G. Andre, Ann. Isr. Phys. Soc. 3, 133 (1980).

[37] Y. E. Kraus, Y. Lahini, Z. Ringel, M. Verbin, and O. Zilberberg, Phys. Rev. Lett. 109, 106402 (2012); Y. E. Kraus and O. Zilberberg, ibid. 109, 116404 (2012); M. Verbin, O. Zilberberg, Y. E. Kraus, Y. Lahini, and Y. Silberberg, ibid. 110, 076403 (2013).

[38] L.-J. Lang, X. Cai, and S. Chen, Phys. Rev. Lett. 108, 220401 (2012); F. Mei, S.-L. Zhu, Z. M. Zhang, C. H. Oh, and N. Goldman, Phys. Rev. A 85, 013638 (2012).

[39] F. Mei, J.-B. You, D.-W. Zhang, X. C. Yang, R. Fazio, S.-L. Zhu, and L. C. Kwek, Phys. Rev. A 90, 063638 (2014).

[40] P. Marra, R. Citro, and C. Ortix, Phys. Rev. B 91, 125411 (2015).
[41] R. D. King-Smith and D. Vanderbilt, Phys. Rev. B 47, 1651 (1993).

[42] E. J. Mele and P. Král, Phys. Rev. Lett. 88, 056803 (2002); S. Onoda, S. Murakami, and N. Nagaosa, ibid. 93, 167602 (2004).

[43] M. Atala, M. Aidelsburger, M. Lohse, J. T. Barreiro, B. Paredes, and I. Bloch, Nat. Phys. 10, 588 (2014); M. Aidelsburger, M. Atala, M. Lohse, J. T. Barreiro, B. Paredes, and I. Bloch, Phys. Rev. Lett. 111, 185301 (2013); M. Aidelsburger, M. Atala, S. Nascimbene, S. Trotzky, Y.-A. Chen, and I. Bloch, ibid. 107, 255301 (2011).

[44] J. Sebby-Strabley, M. Anderlini, P. S. Jessen, and J. V. Porto, Phys. Rev. A 73, 033605 (2006); J. Sebby-Strabley, B. L. Brown, M. Anderlini, P. J. Lee, W. D. Phillips, J. V. Porto, and P. R. Johnson, Phys. Rev. Lett. 98, 200405 (2007).

[45] S. Fölling, S. Trotzky, P. Cheinet, M. Feld, R. Saers, A. Widera, T. Müller, and I. Bloch, Nature (London) 448, 1029 (2007); S. Trotzky, P. Cheinet, S. Fölling, M. Feld, U. Schnorrberger, A. M. Rey, A. Polkovnikov, E. A. Demler, M. D. Lukin, and I. Bloch, Science 319, 295 (2008).

[46] O. Mandel, M. Greiner, A. Widera, T. Rom, T. W. Hansch, and I. Bloch, Phys. Rev. Lett. 91, 010407 (2003); P. J. Lee, M. Anderlini, B. L. Brown, J. Sebby-Strabley, W. D. Phillips, and J. V. Porto, ibid. 99, 020402 (2007).

[47] D. Jaksch and P. Zoller, New J. Phys. 5, 56 (2003); E. J. Mueller, Phys. Rev. A 70, 041603 (2004).

[48] F. Gerbier and J. Dalibard, New J. Phys. 12, 033007 (2010); L. Mazza, A. Bermudez, N. Goldman, M. Rizzi, M. Angel MartinDelgado, and M. Lewenstein, ibid. 14, 015007 (2012).

[49] H. Miyake, G. A. Siviloglou, C. J. Kennedy, W. C. Burton, and W. Ketterle, Phys. Rev. Lett. 111, 185302 (2013).

[50] See the reviews, J. Dalibard, F. Gerbier, G. Juzeliūnas, and P. Öhberg, Rev. Mod. Phys. 83, 1523 (2011); V. Galitski and I. B. Spielman, Nature (London) 494, 49 (2013); N. Goldman, G. Juzeliūnas, P. Öhberg, and I. B. Spielman, Rep. Prog. Phys. 77, 126401 (2014); H. Zhai, ibid. 78, 026001 (2015); S. Zhang, W. S. Cole, A. Paramekanti, and N. Trivedi, arXiv:1411.2297.

[51] K. Jiménez-García, L. J. LeBlanc, R. A. Williams, M. C. Beeler, C. Qu, M. Gong, C. Zhang, and I. B. Spielman, Phys. Rev. Lett. 114, 125301 (2015); Y.-J. Lin, R. L. Compton, K. JiménezGarcía, W. D. Phillips, J. V. Porto, and I. B. Spielman, Nat. Phys. 7, 531 (2011).

[52] J. K. Pachos, E. Alba, V. Lahtinen, and J. J. Garcia-Ripoll, Phys. Rev. A 88, 013622 (2013); D.-W. Zhang, C.-J. Shan, F. Mei, M. Yang, R.-Q. Wang, and S.-L. Zhu, ibid. 89, 015601 (2014).

[53] N. Gemelke, X. Zhang, C.-L. Hung, and C. Chin, Nature (London) 460, 995 (2009); W. S. Bakr, J. I. Gillen, A. Peng, S. Folling, and M. Greiner, ibid. 462, 74 (2009).

[54] C. D. Fertig, K. M. O'Hara, J. H. Huckans, S. L. Rolston, W. D. Phillips, and J. V. Porto, Phys. Rev. Lett. 94, 120403 (2005); N. Strohmaier, Y. Takasu, K. Günter, R. Jördens, M. Köhl, H. Moritz, and T. Esslinger, ibid. 99, 220601 (2007). 